\title{
Un análisis del concepto de familia monoparental a partir de una investigación sobre núcleos familiares monoparentales
}

\author{
Carmen Rodríguez Sumaza \\ Universidad de Valladolid. Departamento de Sociología y Trabajo Social \\ Tomasa Luengo Rodríguez \\ Universidad de Valladolid. Departamento de Psicología
}

\section{Resumen}

El presente artículo busca profundizar en una de las cuestiones más complejas y controvertidas pero quizás menos debatidas de la monoparentalidad: el propio concepto de familia monoparental. Para ello se parte de una definición inicial de familia monoparental que, obtenida tras una revisión del marco teórico existente, sirvió tanto de instrumento de análisis de la monoparentalidad en el marco de una investigación sobre núcleos familiares monoparentales en Castilla y León, como de hipótesis de partida para la explicación de dicho fenómeno. A continuación, se expone el marco metodológico en el que dicho concepto fue sometido a prueba y se revisa la medida en que los rasgos más significativos del concepto reflejaron la realidad de la monoparentalidad en dicha comunidad autónoma. Finalmente, se hace una reflexión sobre el concepto empleado y se apuntan las principales limitaciones que presenta, en un intento de contribuir a la mejora del concepto como herramienta del análisis científico.

Palabras clave: familia monoparental, evaluación de conceptos.

Abstract: An analysis of the concept of lone parent family from an investigation on lone parent family units

The purpose of this paper is to deepen in one of the most complex and less debated questions of loneparethood, the concept of lone parent family itself. We begin with a definition that, obtained from a review of the literature, was used in an empirical research on lone parent families in Castilla y León. Next, we explain the methodological framework in which the concept was tested and review the features of the concept that best captured the reality of lone parent families in that region. Finally, we discuss the results and point to its most important limitations with the goal of improving the concept as a tool for scientific analysis.

Key words: Lone parent family, concept evaluation. 


\section{Sumario}

\section{Introducción}

2. La investigación sobre núcleos familiares monoparentales en Castilla y León

3. Las familias monoparentales como objeto de estudio: algunas dificultades para la identificación y acotación del grupo

4. El proceso de búsqueda e identificación de los rasgos de la monoparentalidad

5. El concepto operativo de familia monoparental empleado en la investigación
6. El concepto como hipótesis de trabajo y el proceso de investigación como proceso de evaluación

7. El concepto de familia monoparental a la luz de los rasgos de la monoparentalidad en Castilla y León

8. Consideraciones finales

Bibliografía

\section{Introducción}

Uno de los cambios más significativos experimentados por las estructuras familiares en España en los últimos años lo constituye el importante incremento del número de hogares compuestos por al menos un núcleo familiar monoparental y del número de personas residiendo en hogares con dichas características. Este fenómeno se enmarca en un proceso de creciente pluralización de las estrategias de convivencia de los españoles. Por un lado, persiste el modelo tradicional de familia nuclear conyugal, constituida por un matrimonio con o sin descendencia. Este modelo familiar no sólo sigue siendo predominante, sino que sigue constituyendo un modelo mayoritario de referencia para buena parte de la población. Por otro lado, junto al papel hegemónico de la familia nuclear, se asiste también a una creciente diversificación de fórmulas alternativas de convivencia, como son, entre otras, los hogares unipersonales, los hogares sin núcleo familiar y los hogares que albergan un núcleo monoparental, todo ello a costa de una disminución en el número de hogares complejos.

En este contexto, el fenómeno de la monoparentalidad ha venido acaparando progresivamente el interés de los estudiosos de la familia en nuestro país en las dos últimas décadas. La mayoría de los análisis practicados, apoyados en la explotación de fuentes estadísticas oficiales (censos de población, encuestas de población activa) o encuestas especiales sobre familias, trabajo, usos del tiempo, hogares pobres, etc., han ido aportando información valiosa y necesaria sobre el volumen, las características sociodemográficas de los progenitores y sus hijos y sus condiciones de vida. Otros trabajos han analizado también los escenarios ideológicos y sociales que han rodeado y rodean la monoparentalidad y las respuestas públicas existentes para resolver las demandas y necesidades de estas familias. Con todo ello no se podría aún decir que el grado de conocimiento de que se dispone en la actualidad sobre la realidad de las familias monoparentales en España sea ni suficiente ni similar al existente en otros países de nuestro entorno socioeconómico, donde, seguramen- 
te debido a que el fenómeno de la monoparentalidad es más antiguo y frecuente, existe no sólo una importante tradición en el análisis de sus múltiples dimensiones, sino que existe además un amplio debate social y político sobre el mismo, como es el caso del Reino Unido o los países nórdicos.

El trabajo que a continuación se presenta pretende retomar una de las cuestiones más complejas y controvertidas pero quizás menos debatidas de la monoparentalidad, el propio concepto de familia monoparental. Son muchos los autores que resaltan en sus trabajos los problemas de definición y alcance del término (Iglesias de Ussel, 1998), la dificultad de partida que supone el hecho de que no exista una definición única y clara de familia monoparental (Moreno Mínguez, 2000), la confusión existente entre los conceptos de familia, hogares y núcleos monoparentales (Durán, 1988; Almeda y Flaquer, 1995), las dificultades de conceptualizar una realidad compleja (Fernández Cordón y Tobío Soler, 1998; Rodríguez Sumaza y Luengo Rodríguez, dirs., 2000), el alto grado de imprecisión del concepto (Fernández Cordón y Tobío Soler, 1999) o que planteen incluso que el concepto de familia monoparental no constituye una categoría adecuada para el análisis del fenómeno (Lefaucheur, 1988). Se echa sin embargo de menos un ejercicio más amplio de reflexión sobre la propia capacidad de los conceptos habituales de "familia monoparental» para servir de instrumento de análisis de la monoparentalidad. Este ejercicio es relevante, pues, como apunta Rodríguez Sumaza (2001a y 2001b), entender cómo está definida públicamente la monoparentalidad puede ayudar, entre otras cuestiones, a comprender mejor la controvertida presencia del tema en los debates públicos y, en última instancia, el nivel de apoyo a estas familias.

Decía Weber que al interpretar las acciones de los sujetos hemos de ser conscientes de que "aquellos conceptos empleados tanto por el lenguaje cotidiano como por el de los juristas (y también por el de otros profesionales), son representaciones de algo que, en parte, existe y, en parte, se presenta como un deber ser en la mente de hombres concretos [...], la acción de los cuales orientan realmente» (Weber, 1969: 12-13). Al hilo de esta afirmación, cabe preguntarse ¿hasta qué punto los conceptos que manejamos son reflejo de algo que tiene entidad por sí mismo?, ¿hasta qué punto son construcciones artificiales diseñadas a modo de instrumentos analíticos para facilitar la comprensión de los hechos, pero que a la vez condicionan su aproximación? El presente trabajo tiene como objetivo el análisis de un concepto de familia monoparental a partir del estudio de las dimensiones más significativas de la monoparentalidad detectadas en el transcurso de una investigación realizada sobre núcleos familiares monoparentales en Castilla y León.

El ejercicio que inicialmente se plantea no está exento de dificultades, pero para redimensionar sus pretensiones es importante insistir en el hecho de que no se busca tanto ofrecer un concepto alternativo a los habitualmente empleados en la literatura científica, como identificar y profundizar en algunas de las limitaciones de los mismos a la hora de reflejar una realidad compleja y diversa. Somos conscientes de que los conceptos no son la realidad, y eso es algo que no les podemos echar en cara. Simplemente consideramos que la reflexión sobre 
las debilidades de los conceptos constituye un mecanismo para la mejora de estos instrumentos básicos del discurso científico.

La necesidad de reflexionar específicamente sobre el concepto de familia monoparental surge al hilo de los trabajos realizados en el marco de un proyecto de investigación sobre núcleos familiares monoparentales en Castilla y León. Como dicha investigación constituye el contexto a partir del cual se procede a la revisión del concepto, el artículo comienza describiendo muy brevemente lo que fueron sus objetivos principales.

Los dos siguientes apartados se centran en las dificultades de acotar a las familias monoparentales como objeto de estudio y el procedimiento empleado para elaborar una definición operacional de familia monoparental a partir del análisis de los rasgos de la monoparentalidad deducidos de las definiciones más habituales encontradas en la bibliografía científica existente en el contexto español en el momento en que se llevó a cabo la investigación.

El concepto de familia monoparental resultante, que fue empleado en el citado estudio, es analizado a continuación a partir de las conclusiones derivadas del proceso de investigación y del propio contacto con los padres y las madres que fueron entrevistados. Para ello, en el apartado sexto se presenta el concepto como una hipótesis de trabajo y el proceso de investigación como un proceso de evaluación. Las limitaciones del concepto que fueron progresivamente detectándose son expuestas finalmente en el epígrafe 7.

$\mathrm{El}$ análisis practicado nos lleva a concluir que el concepto de partida, que tenía la virtud de integrar las dimensiones de la monoparentalidad más habitualmente consideradas por los estudiosos del tema y que nos permitió ciertamente aproximarnos a las estructuras familiares monoparentales, presenta también importantes limitaciones. El proceso de investigación puso en evidencia que el fenómeno de la monoparentalidad es ciertamente complejo y poliédrico. Difícilmente se puede hablar así de la familia monoparental como un tipo posible de forma familiar, cuando en la realidad nos encontramos con diferentes tipos de familias monoparentales como consecuencia de que las personas acceden a la monoparentalidad por vías muy diversas, disponen de aprendizajes sociales diferentes, son distintas las situaciones sociales a las que a diario se enfrentan y es plural el significado que los hechos tienen para sus miembros.

Tras la denominación simplificadora de familia monoparental se esconde un panorama complejo y diverso de estrategias familiares que probablemente tienen menos cosas en común que las que comparten (diferentes experiencias, identidades, contenidos, situación socioeconómica, relaciones, etc.). Todo ello constituye una verdadera invitación a revisar, o cuando menos a discutir, los conceptos habituales de familia monoparental. Esta cuestión refuerza la idea de que resulta preciso hacer investigaciones específicas de cada subtipología de familia monoparental para conseguir un conocimiento más profundo del fenómeno de la monoparentalidad, lo que constituye todo un reto para la sociología de la familia (Mora, 2001). 


\section{La investigación sobre núcleos familiares monoparentales en Castilla y León}

El análisis del concepto de familia monoparental que aquí se presenta surge de la necesidad de retomar una serie de interrogantes surgidos a partir de una investigación sobre núcleos familiares monoparentales en Castilla y León ${ }^{1}$. Este trabajo constituye, pues, el contexto a partir del cual se procede a la revisión del concepto de familia monoparental, por lo que a continuación se explica lo que fueron sus objetivos principales.

La investigación se planteó como objetivo central el análisis de la situación actual y las características de los individuos integrantes de núcleos familiares monoparentales en una comunidad autónoma donde, en sintonía con lo ocurrido a nivel nacional y europeo, se asistía a un auge de estas estrategias de convivencia, si bien a un ritmo más moderado. Hay que señalar que el fenómeno de la monoparentalidad en Castilla y León fue interpretado en el marco de los cambios ocurridos en las estructuras familiares en las últimas décadas en España y en el resto de Europa, realizándose una estimación cuantitativa de la importancia de estas formas familiares en la comunidad autónoma de referencia.

En el estudio se analizaron cuestiones tan variadas y complejas como son la propia naturaleza de la monoparentalidad en la región, la estructura de los hogares monoparentales, los contenidos relacionales de este tipo de familias, la importancia del hogar como espacio básico de la vida en familia, el nivel educativo y la situación laboral de los adultos al frente de los núcleos monoparentales, la situación económica del grupo, la disponibilidad de tiempo libre, el grado de asociacionismo y de participación ciudadana de los progenitores y la capacidad ordinaria de las unidades familiares monoparentales para dar cobertura a las distintas necesidades que el grupo y sus miembros tienen. Se analizó también la percepción de los progenitores acerca de las principales ventajas y dificultades derivadas de la situación de monoparentalidad.

En el difícil intento de conocer y explicar los comportamientos, los significados y las experiencias subjetivas de los padres y madres, se diseñó una metodología de recogida de información de carácter eminentemente cualitativo, si bien se combinaron técnicas cualitativas y cuantitativas. El análisis practicado primó el estudio de la vida cotidiana tomando como unidad de referencia el individuo y prestó una atención fundamental a lo que pensaban y opinaban los protagonistas, al tiempo que se recurrió también al estudio de sus comportamientos. Se buscó fundamentalmente con ello analizar, en primer lugar, la experiencia subjetiva de la monoparentalidad vivida por estos padres y madres en Castilla y León y, a partir de esto, comprender y explicar su experiencia en base a la información que ellos mismos proporcionaron. También se intentó hacer una lectura de algunas de sus prácticas más habituales, como la forma

1. Los resultados del trabajo mencionado se pueden encontrar en RoDRíGUEZ SUMAZA, C. y LUENGO RodríGUEZ,T. (dirs.) (2000). 
en que se estructuraban los hogares monoparentales o algunas de las estrategias emprendidas por el grupo (de carácter económico, residencial, social, etc.) y que eran empíricamente observables.

\section{Las familias monoparentales como objeto de estudio: algunas dificultades para la identificación y acotación del grupo}

La delimitación clara y rigurosa de lo que se va a considerar objeto de análisis en una investigación social tiene una importancia decisiva respecto al valor del estudio en cuestión. En un estudio sociológico la unidad básica de análisis es el grupo identificado y, para poder identificar un grupo, es requisito previo definirlo y acotarlo lo más precisa y operativamente posible.

En el caso concreto del trabajo realizado sobre núcleos familiares monoparentales en Castilla y León, el grupo objeto de estudio era la familia monoparental. La tarea de identificación de dicho grupo no era fácil, y ello por varias razones. En primer lugar, porque el propio objeto genérico de análisis -la familia - es una realidad dinámica y compleja. Resulta cambiante porque con el tiempo varían tanto las formas como los contenidos de lo que se entiende por familia. Y es también multifacética, al ser muchos los aspectos que configuran el panorama familiar (relacionales, culturales, políticos, económicos, jurídicos, religiosos, personales, etc.). Asimismo, nos encontramos ante una institución - la familia - cuyos contenidos varían no sólo con el tiempo, sino también con el espacio, existiendo una gran diversidad de modelos familiares en cada sociedad y en cada cultura. Es debido fundamentalmente a estas cuestiones que no existe un concepto único y universal de familia (y por tanto de familia monoparental) que permita incorporar o integrar la rica variedad de relaciones, contenidos, estructuras, experiencias y funciones que caracterizan a los sistemas familiares.

En el intento de acotar a las familias monoparentales como objeto de estudio se añaden a las dificultades mencionadas otras circunstancias adicionales. Estamos, como se verá más adelante con detalle, ante un grupo que experimenta procesos y situaciones muy variadas y que no solamente son vividos de muy distinta manera por los propios sujetos que los protagonizan, sino que han sido y son interpretados de muy diferente modo por los colectivos y las sociedades a las que éstos pertenecen (McIntosh, 1996; Lewis, 1999). Hasta hace bien poco tiempo las distintas situaciones familiares monoparentales recibían un tratamiento social muy diferente. Así, frente al respeto, la protección social y un cierto prestigio social de los que frecuentemente han disfrutado las viudas, las madres solteras, en cambio, han sido objeto frecuente de desprecio y discriminación.

Otro elemento que introduce una especial dificultad en la acotación de la monoparentalidad es su propia temporalidad. Como señalan Fernández Cordón y Tobío Soler (1998) a partir de las investigaciones realizadas por Martín (1994) y Duncan y otros (1994), una parte importante de la población experimenta a lo largo de su vida diversas situaciones de monoparentalidad, tanto 
como hijo o como progenitor, pero que generalmente representan una fase de trayectorias en las que el individuo se integra en diferentes tipos de familia. Nos encontramos así con que no todas las estructuras familiares monoparentales tienen un carácter necesariamente permanente ${ }^{2}$. Quiere esto decir también que cualquier análisis de corte transversal que se practique para analizar el fenómeno de la monoparentalidad sólo permitirá captar, en el mejor de los supuestos, los casos existentes a la fecha de referencia. Esta cuestión ciertamente no es exclusiva del estudio de las familias monoparentales, pero no por ello hay que dejar de mencionarla.

En síntesis, la acotación de las familias monoparentales como objeto de estudio adolece de entrada de una especial complejidad al integrar situaciones familiares y sociales muy diversas, algunas de las cuales siempre han existido (madres solteras, progenitores viudos), mientras que otras son más nuevas (padres/madres separados, divorciados o adoptivos), presentando todas ellas una importancia relativa variable en estos últimos años.

\section{El proceso de búsqueda e identificación de los rasgos de la monoparentalidad}

Con el fin de delimitar el objeto de estudio, las familias monoparentales, se partió en un primer momento de una serie de definiciones procedentes de investigaciones existentes en el contexto español, en la confianza de que recurriendo al método comparativo se estaba en mejores condiciones para identificar e integrar los rasgos más habitualmente considerados por otros autores como propios de estas familias.

A continuación se ofrece un cuadro que contiene algunas de las principales definiciones de familia monoparental encontradas en la literatura revisada y que sirvieron de punto de partida de este intento de acotar el objeto de la investigación (cuadro 1). Somos conscientes de que en el cuadro se recoge sintética y estrictamente lo que fue presentado por cada autor como definición del concepto, con lo que se pierden las aportaciones complementarias a la estricta definición que a lo largo de la obra de cada autor pueden encontrarse. No obstante, esta síntesis ofrece la ventaja de ofrecer una visión panorámica inicial del objeto genérico de estudio en base a investigaciones previas.

A partir de las diferentes definiciones encontradas, se realizó un estudio comparativo para detectar las diferencias entre ellas y, fundamentalmente, las características más frecuentes. En el cuadro 2 se presenta un esquema de los principales rasgos que los autores estudiados habían empleado para caracterizar

2. Algunos estudios realizados en el Reino Unido han constatado que una proporción relevante de familias monoparentales cambia de estructura familiar en menos de cinco años (Ford, Marsh y Mckay, 1995; ESRC, 1994, y Noble, Smith y Cheung, 1998), estando la duración media de la estructura monoparental en estrecha relación con las características personales de los progenitores (estado civil, edad, nivel de estudios, número de hijos, etc.) (Ermish, Jenkins y Wright, 1990). 


\section{Cuadro 1. Algunas definiciones de familia monoparental}

\section{Autor/es}

Consejo de Europa

(1995)

Alberdi, I.

(1988: 101)

Borrajo Iniesta, $\mathrm{S}$.

(1988: 43)

\section{Definición}

Toda familia constituida por un solo progenitor y uno o más hijos.

Familia encabezada por una sola persona adulta, hombre o mujer, y en la que hay más miembros que dependen económicamente y socialmente de ella.

Familia formada por un adulto que vive sólo con uno o más hijos a su cargo y que en su formación ha de haber seguido una de las tres vías siguientes:

- Fallecimiento en un matrimonio con hijos pequeños de uno de los cónyuges.

- Ruptura de la pareja con hijos menores por conflicto entre sus miembros, quedando los hijos en la custodia de uno de los padres.

- Madre soltera con uno o más hijos nacidos fuera del matrimonio.

Duran, $M^{a}$ A.

(1988: 16)

Hogares en los que un solo adulto asume por necesidad el cuidado de sus hijos menores de edad.

Iglesias de Ussel, J. (1988a: 28)

Convivencia de un solo miembro de la pareja —varón o mujer-con hijos no emancipados.

Le Gall, D. y Martín, C. Hogares compuestos por una persona (hombre o mujer) que vive sola con uno (1988: 195) o más niños.

Sayn, I.

(1988: 203)

Las formadas por un solo progenitor responsable directo de la custodia de los menores.

Comisión Europea

Progenitor que sin convivir con su cónyuge ni cohabitando con otra persona, convive al menos con un hijo dependiente y soltero.

Roll, J.

(1992: 160-161)

Un padre o madre que no vive en pareja (entendiendo pareja casada o que cohabite). Puede vivir o no con otras personas (amigos, padres) y vive al menos con un hijo menor de 18 años (distinto de hijo dependiente).

El término "hijo dependiente» implica que el hijo todavía sigue siendo educado en algún sentido, pero también que es económicamente dependiente.

Almeda, E. y Flaquer, L. La configuración formada por un progenitor (padre o madre) con alguno de (1995: 26) sus hijos solteros.

Un núcleo familiar monoparental puede constituir en si un hogar independiente (un hogar monoparental) o bien puede estar formado de un hogar más amplio en el que residen otros núcleos o parientes.

Iglesias de Ussel, J.

(1998: 237)

Situación familiar de convivencia de uno o de varios hijos menores - generalmente menores de 18 años - con uno sólo de sus progenitores, sea el padre o la madre, por cualquier causa.

Comisión de los Derechos de la Mujer (1998)
Los estudios revelan una imagen sumamente compleja y variada de estructuras sociales y de ayuda para los hijos y el progenitor solo, demasiado diversas entre sí como para crear una imagen homogénea. La familia monoparental puede tener su origen en situaciones muy diversas. En la mayoría de los casos el progenitor solo se encuentra en una situación muy vulnerable, teniendo que hacer frente a responsabilidades por partida doble en calidad de proveedor del sustento y cuidador de la familia. 
Cuadro 2. Características presentes en algunas definiciones de familia monoparental

\section{Características de las familias monoparentales}

\begin{tabular}{|c|c|c|c|c|c|c|c|c|c|c|}
\hline Autores & $\begin{array}{l}\text { Un solo } \\
\text { progenitor }\end{array}$ & $\begin{array}{c}\text { Edad } \\
\text { progenitor }\end{array}$ & $\begin{array}{c}\text { Sexo } \\
\text { progenitor }\end{array}$ & $\begin{array}{l}\text { Presencia } \\
\text { hijos }\end{array}$ & $\begin{array}{l}\text { Edad } \\
\text { hijos }\end{array}$ & $\begin{array}{l}\text { Estado civil } \\
\text { hijos }\end{array}$ & $\begin{array}{l}\text { Dependencia } \\
\text { hijos }\end{array}$ & $\begin{array}{c}\text { Tipo de } \\
\text { dependencia }\end{array}$ & $\begin{array}{c}\text { Convivencia } \\
\text { con otras } \\
\text { personas }\end{array}$ & $\begin{array}{c}\text { Heterogeneidad } \\
\text { en las causas }\end{array}$ \\
\hline $\begin{array}{l}\text { Consejo de Europa } \\
\text { (1985) }\end{array}$ & $x$ & & & $\mathrm{x}$ & & & & & & \\
\hline Alberdi, I. (1988) & $\mathrm{x}$ & $\mathrm{x}$ & $\mathrm{x}$ & $\mathrm{x}$ & & & & $\mathrm{x}$ & & \\
\hline $\begin{array}{l}\text { Borrajo Iniesta, S. } \\
\text { (1988) }\end{array}$ & $\mathrm{x}$ & $\mathrm{x}$ & & $\mathrm{x}$ & $\mathrm{x}$ & & $\mathrm{x}$ & & & $\mathrm{x}$ \\
\hline Durán, Ma A. (1988) & $\mathrm{x}$ & $\mathrm{x}$ & & $\mathrm{x}$ & $\mathrm{x}$ & & & & & $\mathrm{x}$ \\
\hline $\begin{array}{l}\text { Iglesias de Ussel, J. } \\
\text { (1988) }\end{array}$ & $\mathrm{x}$ & & $\mathrm{x}$ & $\mathrm{x}$ & & & $\mathrm{x}$ & & & \\
\hline $\begin{array}{l}\text { Legall, D. y Martín, C. } \\
\text { (1988) }\end{array}$ & $\mathrm{x}$ & & $\mathrm{x}$ & $\mathrm{x}$ & & & $\mathrm{x}$ & & & \\
\hline Sayn, I. (1988) & $\mathrm{x}$ & & & $\mathrm{x}$ & $\mathrm{x}$ & & $\mathrm{x}$ & & & \\
\hline $\begin{array}{l}\text { Comisión Europea } \\
\text { (1989) }\end{array}$ & $\mathrm{x}$ & & & $\mathrm{x}$ & & $\mathrm{x}$ & $\mathrm{x}$ & & $\mathrm{x}$ & \\
\hline Roll, J. (1992) & $\mathrm{x}$ & & $\mathrm{x}$ & $\mathrm{x}$ & $\mathrm{x}$ & & & $\mathrm{x}$ & $\mathrm{x}$ & \\
\hline $\begin{array}{l}\text { Almeda, E. y Flaquer, L. } \\
\text { (1995) }\end{array}$ & $\mathrm{x}$ & & & $\mathrm{x}$ & & $\mathrm{x}$ & & & $\mathrm{x}$ & \\
\hline $\begin{array}{l}\text { Iglesias de Ussel, J. } \\
\text { (1998) }\end{array}$ & $\mathrm{x}$ & & $\mathrm{x}$ & $\mathrm{x}$ & $\mathrm{x}$ & & & & & $\mathrm{x}$ \\
\hline $\begin{array}{l}\text { Comisión de los } \\
\text { Derechos de la Mujer } \\
\text { (1998) }\end{array}$ & $\mathrm{x}$ & & & $\mathrm{x}$ & & & $\mathrm{x}$ & & $\mathrm{x}$ & $\mathrm{x}$ \\
\hline
\end{tabular}


a las familias monoparentales. Es importante insistir en el hecho de que en la mayoría de las obras de las que fueron extraídas las definiciones ofrecidas pueden llegar a descubrirse gran parte de otros rasgos posibles, por lo que podría llevar a error suponer que determinados autores no contemplan algunas características que no aparecen de forma explícita dentro del concepto. Esta es sin duda una limitación propia de todo ejercicio de síntesis. Pero también hay que tener presente que cuando un autor ha querido definir la realidad compleja de la familia monoparental, ha practicado una determinada selección de rasgos que ha considerado como más relevantes, y éstos son los que nosotros hemos analizado.

A la vista del análisis practicado, los elementos más significativos de las distintas definiciones de familia monoparental manejadas resultaron ser los siguientes:

\section{La presencia de un solo progenitor en el hogar familiar}

La no convivencia en pareja parece ser una de las condiciones fundamentales para que una familia pueda ser considerada como monoparental. Existe consenso casi absoluto entre los autores revisados en que el grupo se organiza en torno a un solo progenitor, que puede ser hombre o mujer, y en la mayoría de los casos adulto.

Pocas definiciones asumen explícitamente que este progenitor pueda compartir espacio o cohabitar con otras personas sin que el grupo familiar pierda su condición de monoparentalidad (Rol, 1992; Almeda y Flaquer, 1995). Domina, pues, la idea de que cuando junto al núcleo monoparental convive la nueva pareja del progenitor estamos ante una de las situaciones que da por finalizada la monoparentalidad, pasando a configurar lo que se conoce como «familia reconstituida» (Iglesias de Ussel, 1998: 237).

\section{La presencia de uno o varios hijos en el hogar familiar}

Todas las definiciones estudiadas completan el grupo familiar con uno o varios hijos a cargo del adulto responsable de la familia. La presencia viene a definirse en términos físicos, resultando por tanto necesaria la corresidencia habitual en el hogar familiar.

\section{La dependencia de los hijos}

Otro elemento fundamental del concepto de monoparentalidad es la carga o dependencia de los hijos respecto del adulto, implícita en todas las definiciones recogidas. Las diferencias se refieren a ciertos criterios que van a definir esa dependencia, entre ellos la edad y el estado civil de los hijos. Lo más frecuente es definir una edad por debajo de la cual se considera que todos los hijos son dependientes. En la mayor parte de los trabajos revisados esta edad se sitúa en torno a la mayoría de edad (los 18 años en el caso español), excepto en aquéllos que elevan la edad recurriendo a la idea de "hijos no emancipados», ampliando el grupo hasta los 25 años.

En esta última línea se manifiestan Almeda y Flaquer (1995) justificando la dependencia por la función socializadora que cumple todo grupo familiar, y 
señalando que «la maduración cada vez más tardía de los jóvenes debido a la prolongación de sus estudios, a su difícil inserción en el mercado de trabajo y a la crisis de la vivienda aconsejan elevar ese límite hasta los 25 años». Otro matiz importante en la línea iniciada en 1989 desde la Comisión Europea la aportan estos autores, al introducir la condición de que los hijos sean solteros.

\section{Heterogeneidad en las causas que derivan la monoparentalidad}

Por último, la complejidad de la monoparentalidad viene dada por la diversidad de circunstancias que en las sociedades actuales pueden generar familias monoparentales. A situaciones que vienen de antiguo - como la procreación fuera del matrimonio, la viudedad o la separación de la pareja por motivos laborales, enfermedad o privación de libertad- se suman situaciones nuevas, consecuencia de las separaciones conyugales y el divorcio. Emergiendo con fuerza asistimos también en la actualidad a un nuevo tipo de monoparentalidad derivada del marco legal: la adopción por parte de un solo adulto.

Esta diversidad de circunstancias origina familias muy heterogéneas en cuanto a su naturaleza y a su desarrollo cotidiano. En este sentido, la tipología propuesta por Iglesias de Ussel (1988: 28) es clarificadora, pues permite clasificar las principales circunstancias que dan lugar a la monoparentalidad en las cuatro categorías siguientes:

a) Monoparentalidad vinculada a la natalidad, circunstancia que derivará los núcleos monoparentales de las madres solteras.

b) Monoparentalidad vinculada a la relación matrimonial, categoría que acoge el gran abanico de situaciones de ruptura voluntaria e involuntaria de la pareja.

c) Monoparentalidad vinculada al ordenamiento jurídico, tipología que acoge la maternidad y paternidad social a través de los procesos de adopción.

d) Monoparentalidad vinculada a situaciones sociales y cuya naturaleza se fundamenta en la ausencia de uno de los cónyuges por motivos de trabajo, privación de libertad, enfermedades prolongadas y emigración.

\section{El concepto operativo de familia monoparental empleado en la investigación}

A partir de la revisión de los rasgos de la monoparentalidad considerados como fundamentales en las definiciones revisadas, teniendo en cuenta también los propios objetivos del proyecto y la información procedente de la fase exploratoria del estudio, las familias monoparentales quedaron definidas en términos de la investigación en Castilla y León de la siguiente manera:

Familia monoparental es todo núcleo familiar constituido por un hombre o una mujer viviendo al menos con uno o varios hijos menores de 18 años a su cargo o que, superando esa edad pero siendo menores de 26 años, presenten alguna circunstancia o algún tipo de minusvalía que haga que la relación de dependencia en sus aspectos instrumentales se mantenga. 
La población objeto de estudio fue definida así como el «conjunto de los padres y madres que no viven en pareja pero conviven con hijos e hijas dependientes a su cargo, y residentes en los ámbitos rural, semiurbano y urbano de las nueve provincias de la Comunidad Autónoma de Castilla y León». La definición operacional de familia monoparental exigía de este modo que, para que un grupo familiar pudiera ser considerado como un caso de monoparentalidad, éste satisfaciera los criterios siguientes:

\section{A. Al frente del núcleo monoparental debia haber únicamente un progenitor}

Esta circunstancia, desde un punto de vista formal, puede ser el resultado de:

A.1. Un proceso de cambio familiar que obliga a una reestructuración de la unidad familiar por:

- Ausencia definitiva derivada del fallecimiento de uno de los miembros de la pareja.

- Ausencia derivada de la ruptura de la pareja.

- Ausencia temporal ocasionada por motivos laborales, de salud o por la encarcelación de uno de los progenitores.

A.2. Un acceso a la monoparentalidad vía la maternidad/paternidad directa, biológica (madres solteras) o por adopción.

B. La adultez del progenitor

Sólo se consideraron los padres y madres mayores de edad.

C. La presencia en el núcleo familiar de uno o varios hijos.

D. La dependencia de los hijos respecto del progenitor custodio

Los límites de la dependencia fueron acotados por la edad de los hijos, su situación económica y sus capacidades. Así, se consideró que los hijos son dependientes en los casos siguientes:

D.1. Hijos menores de 18 años, con independencia de sus capacidades físicas e intelectuales.

D.2. Hijos entre 18 y 26 años cuando éstos padezcan algún tipo de minusvalía que prolongue la dependencia del padre o de la madre en los aspectos instrumentales.

D.3. Hijos entre 18 y 26 años con plena capacidad física e intelectual que dependan económicamente de su padre o su madre.

\section{E. La posible convivencia del núcleo monoparental con otras personas}

Se optó por entender que una familia monoparental es un núcleo familiar monoparental, el cual puede residir independientemente, constituyendo entonces un hogar monoparental, o compartiendo el lugar de residencia con otras personas (familiares o no) en un hogar múltiple o extenso. 


\section{F. La heterogeneidad en las causas que derivan en monoparentalidad \\ Se asumieron todas las circunstancias que puedan dar lugar al surgi- miento de un núcleo monoparental.}

\section{G. Lugar de residencia}

Se estudiaron familias monoparentales residiendo a la fecha de referencia tanto en viviendas familiares como en viviendas colectivas (casas de acogida, etc.) en el territorio geográfico de Castilla y León.

La definición operacional del grupo familiar que acabamos de exponer fue la unidad de referencia básica para el estudio de la monoparentalidad en Castilla y León. Es, por tanto, el concepto central del estudio que se sometió a prueba con cada una de las técnicas aplicadas y en cada una de las fases de la investigación, como a continuación se expondrá.

\section{El concepto como hipótesis de trabajo y el proceso de investigación como proceso de evaluación}

Los conceptos, entendidos como herramientas de análisis de una realidad, tienen la virtud de permitir acercarnos a conocer los hechos. Presentan sin embargo una importante limitación, la de deformar la realidad a la que hacen referencia a fuerza de simplificar sus complejas dimensiones y adaptarse al campo de visión del observador. Así, como los mapas son representaciones a escala del espacio físico, los conceptos son los instrumentos a través de los cuales aprehendemos lo que nos rodea. Su carácter simplificador es por ello tanto un problema como una virtud, al resultar la clave que permite asimilar esa realidad. No se puede pretender que los conceptos sean la propia realidad a la que representan, del mismo modo que no tiene sentido ni utilidad un mapa que sea tan perfecto que ocupe todo el territorio que representa. Sin embargo, sí se puede pedir que los instrumentos que utilizamos para comprender la realidad se perfeccionen, mejoren. Y es obligación del científico revisar críticamente el utillaje empleado, calibrar su calidad con el objetivo de mejorarla.

Para poder evaluar la calidad del concepto operativo de familia monoparental empleado en la investigación sobre núcleos familiares monoparentales en Castilla y León, se trabajó desde un primer momento con dicho concepto como si de una hipótesis de trabajo se tratara. El propio proceso de investigación y el propio contacto con las familias monoparentales serían la prueba de fuego que permitiría detectar las bondades del concepto, así como sus problemas y carencias. Con el objeto de identificar éstos últimos, exponemos a continuación brevemente el método y los procedimientos empleados en la investigación, que se constituyen así en el mecanismo de la evaluación del concepto de partida. Somos no obstante conscientes de que las reconstrucciones racionales de los procesos de investigación, tan necesarias para evaluar el proceder científico, tienen también el peligro de acabar legitimando los pasos dados por el propio investigador, por lo que pretendemos una revisión crítica del proceso. 
La investigación, de naturaleza eminentemente cualitativa, se desarrolló en tres etapas, combinando técnicas cualitativas y cuantitativas. La aproximación a las familias objeto de análisis se realizó en las siguientes fases:

Fase 1. En la primera fase, de naturaleza exploratoria, se explotaron los datos secundarios procedentes de las principales fuentes estadísticas demográficas, y se desarrolló un programa de entrevistas semiestructuradas a una muestra representativa de un conjunto de informantes estratégicos que fueron participando posteriormente en el estudio en todas sus fases.

La combinación de estas técnicas permitió:

1. Estimar cuantitativamente el número de familias en situación de monoparentalidad en Castilla y León y contrastar su importancia relativa en éste y otros ámbitos geográficos.

2. Sensibilizar a los informantes estratégicos en su función mediadora en el estudio.

3. Poner en marcha un plan de comunicación con los agentes sociales relacionados con la monoparentalidad.

Los informantes clave o informantes estratégicos, figura clave en investigaciones de naturaleza cualitativa, tanto como expertos apadrinadores del investigador en el escenario como fuentes de información primaria (Taylor y Gogdan, 1994), permitieron resolver cuestiones decisivas para la estrategia de recogida de información. A través de ellos pudimos acceder a un amplio conjunto de instituciones y organizaciones en contacto con adultos en situación de monoparentalidad y, a través de éstas, a los propios sujetos en situación de monoparentalidad.

En base a los recursos existentes en la comunidad, se montó una red de informantes lo suficientemente amplia, de manera que estuviera representada en ella la heterogeneidad del fenómeno objeto de estudio. Se seleccionó así un amplio abanico de instituciones y organizaciones que nos dieran cuenta de la monoparentalidad en su doble dimensión, dependencia social versus independencia social. El criterio esencial para formar las diversas redes de recogida de datos consistió en que sus componentes estuvieran cerca de las familias en situación de monoparentalidad y, por tanto, tuvieran un probado conocimiento y cierta experiencia acerca de esa realidad social.

Los componentes de la red de informantes estratégicos se clasificaron según su posición y el momento de intervención en el estudio como informantes estratégicos de primer orden e informantes estratégicos de segundo orden. Recibieron la denominación de informantes de primer orden los profesionales directamente relacionados con miembros de familias monoparentales (trabajadores sociales de los centros de acción social, profesionales de los juzgados de familia y juzgados de menores, etc.).

Fueron considerados como informantes de segundo orden los profesionales que, sin estar directamente relacionados con las familias objeto de estudio, 
tenían una posición jerárquica en las instituciones u organizaciones a las que pertenecían que les permitía impulsar y apoyar la obtención de unidades de observación que, por la vía de los informantes de primer orden, pudiera quedar descubierta. En esta segunda categoría quedaron incluidos directores de área y jefes de servicio de diversas instituciones, coordinadores de equipos de acción social, presidentes de asociaciones, etc.

Fase 2. En la segunda etapa, de naturaleza cuantitativa, se aplicó a una muestra de familias monoparentales un cuestionario con 59 preguntas estructuradas que recogían las variables más relevantes en relación con el fenómeno de la monoparentalidad.

$\mathrm{Al}$ no existir una base de sondeo de las familias monoparentales en Castilla y León, es decir, un documento «escrito o informatizado en el que estén consignados todos y cada uno de los elementos del universo" (Ruíz Olabuénaga; Aristegui y Melgosa, 1998: 87), no podía hacerse una extracción probabilística de los elementos. Se optó, por tanto, por una muestra en la que las familias monoparentales no fueran elegidas por las leyes del azar, sino de forma intencional y controlada.

Como consecuencia de ello se recurrió al siguiente procedimiento:

a) Elaboración de una muestra intencional o razonada, obtenida a partir de una selección de informantes clave.

b) Elaboración de una muestra por el procedimiento denominado de bola de nieve. Mediante el mismo, informantes muy especiales nos llevan, por familiaridad, por conocimiento o por facilidad de acceso, a las familias objeto de estudio, que sirven, a su vez, de unidades adicionales de muestreo.

Fase 3. En la tercera fase, de naturaleza cualitativa, se celebraron entrevistas en profundidad a personas expertas en el tema y a madres y padres en situación de monoparentalidad y paneles de expertos con la presencia de profesionales de ámbitos tales como la psicología jurídica, el trabajo social en materia de protección de menores, el tejido asociativo de mujeres y hombres en situación de monoparentalidad, la psicología en el ámbito de la acción social, el derecho de familia y de la seguridad social y el trabajo social en el ámbito de los servicios sociales. Todos ellos dieron significado adicional, desde la vivencia y la práctica cotidiana, al análisis de las variables estudiadas.

\section{El concepto de familia monoparental a la luz de los rasgos de la monoparentalidad en Castilla y León}

Habíamos señalado hasta aquí que, a efectos de la investigación sobre núcleos familiares monoparentales en Castilla y León, entendíamos que una familia monoparental supone la convivencia de un solo progenitor - hombre o mujercon al menos un hijo o una hija dependiente a su cargo. Asimismo, habíamos fijado como objetivo validar la capacidad del concepto de familia monopa- 
rental empleado inicialmente para recoger las dimensiones que el fenómeno presentaba en el ámbito geográfico de referencia.

En relación con los resultados obtenidos en la investigación, hay que decir que, por una parte, se confirmaron de manera consistente los rasgos o dimensiones más significativos del concepto de monoparentalidad expuestos anteriormente, dimensiones observadas asimismo en otros contextos geográficos por numerosos investigadores. Por otro lado, y aquí radica la parte más interesante del proceso de revisión del concepto, fueron evidenciándose nuevos elementos de cara a una mejor comprensión del fenómeno de la monoparentalidad. Ambas cuestiones se exponen a continuación.

$\mathrm{Si}$ revisamos lo que inicialmente fueron consideradas como las dimensiones más fundamentales que definen la monoparentalidad, nos encontramos con los siguientes resultados para Castilla y León:

\section{A. La presencia en el hogar familiar de un único progenitor}

La ausencia mayoritaria del padre en el núcleo monoparental nos lleva a encontrarnos ante una monoparentalidad eminentemente femenina (monomarentalidad), lo que indica que la variable género va a tener un peso importante en la configuración de la naturaleza de la monoparentalidad. En términos de la encuesta realizada, la persona de referencia resultó ser una mujer en el $90,5 \%$ de los casos. Se trata ésta de una característica coincidente con la pauta generalizada en España y en todos los países de nuestro entorno europeo, donde la mayoría de los responsables de este tipo de familias son mujeres.

La exigencia de considerar necesaria la presencia en el hogar familiar de un único progenitor para poder afirmar que estamos ante una familia monoparental plantea, sin embargo, importantes consideraciones. Se comprobó así, en no pocas ocasiones, lo inexacto y forzado que resulta incluir en la misma categoría los siguientes casos:

- Unidades familiares monoparentales consecuencia de un proceso de separación o divorcio donde ambos progenitores comparten las tareas de educación y atención a los hijos, aunque éstos últimos corresidan la mayor parte del tiempo con uno solo de los padres.

- Unidades familiares monoparentales donde, pese a la ausencia física de uno de los progenitores, el grupo familiar mantiene intacta la funcionalidad y no se percibe a sí mismo como diferente al modelo convencional de familia nuclear. Esta situación resultó ser frecuente en los casos de familias donde un progenitor no correside con la familia por razones laborales o por enfermedad.

- Unidades familiares donde un progenitor está ausente o no existe, pero otro adulto, corresidente o no en el hogar, ejerce las funciones que supuestamente debería realizar el progenitor ausente.

La circunstancia de que formalmente haya un único progenitor en el núcleo familiar para que podamos hablar de familia monoparental no debe ser enten- 
dida, por tanto, como que estamos ante un núcleo familiar incompleto, pues los adultos al frente de un núcleo familiar monoparental no son necesariamente padres o madres solos o solas en sentido estricto o con responsabilidades familiares no compartidas.

Las situaciones anteriores nos aproximan a las tesis que insisten en diferenciar la idea de "grupos monoparentales» de la idea de "familias monoparentales» (Lefaucheur, 1988). Desde el punto de vista estructural, una unidad familiar de corresidencia puede no contar con la presencia de uno de los progenitores, pero la noción de familia excede con mucho el ámbito de lo doméstico, y funcional y emocionalmente la familia puede ser bifocal y más similar en sus contenidos a lo que habitualmente suponemos propio de las estructuras familiares nucleares.

Las consideraciones previas también llevan a reflexionar sobre lo insuficiente que resulta el estudio de las familias tomando como principal referente las formas familiares. Las familias, además de grupos, son contenidos. Y resulta obvio que en similares estructuras no tienen por qué presentarse contenidos idénticos, del mismo modo que funciones similares pueden llevarse a cabo en el seno de estructuras diferentes.

Directamente de aquí podemos también derivar lo inexacto de no considerar que familias nucleares conyugales asimétricas, donde las responsabilidades de atención y educación a los hijos recaen exclusivamente en uno de los cónyuges, pueden constituir, desde el punto de vista de los contenidos, unidades familiares monoparentales de facto.

\section{B. La adultez del progenitor}

El supuesto de adultez del progenitor custodio se cumplió ampliamente entre los padres y madres estudiados. La mayoría de ellos (el 57,7\%) se encontraba entre los 26 y los 40 años de edad. Los más numerosos (el 42,8\%) tenían entre 31 y 40 años, hasta alcanzar los 50 años el número de encuestados representó el 25,4\% de la muestra, y por encima de esta edad el número de encuestados representó sólo el 9,3\%. En el otro extremo, un 7,3\% fueron menores de 26 años, en su mayoría madres solteras.

Aunque efectivamente la mayoría de los progenitores superan la edad de los 18 años, es importante señalar que fue lógicamente constatada la existencia de madres adolescentes. Aunque minoritaria desde el punto de vista cuantitativo, esta realidad merece ciertamente ser analizada con detalle, pues constituye un grupo con unas características, necesidades y situaciones familiares verdaderamente singulares, como señala Delgado (1994). La especial complejidad de este grupo y el insuficiente conocimiento existente sobre el mismo, bien exigen su no exclusión del conjunto de familias monoparentales, como en ocasiones es habitual.

Se constató también la existencia de casos en los que el adulto al frente del grupo monoparental no es exactamente un progenitor, sino otro adulto, casi siempre con alguna vinculación consanguínea con los menores (por ejemplo abuelos o familiares cercanos). Esta circunstancia tampoco fue analizada por no caber en la definición operacional de partida. 


\section{La presencia en el núcleo familiar de uno o varios hijos}

El número de hijos más frecuente en el seno de las familias monoparentales castellano-leonesas fue de uno. Esto ocurrió en un 56,7\% de las familias encuestadas, mientras que dos hijos había en el 24,7\% y más de tres tan sólo en el $9 \%$. Las familias con mayor número de hijos fueron las encabezadas por viudos y viudas y las procedentes de una ruptura de pareja.

La exigencia de la presencia de hijos en el núcleo familiar también plantea importantes consideraciones. Es importante señalar en este sentido la reivindicación de ciertos padres y madres cuyos hijos no corresiden la mayoría del tiempo con ellos, pero que comparten las tareas de educación y atención a los hijos con el progenitor custodio y que conviven periódica y sistemáticamente con sus hijos, de ser considerados también como unidades familiares de plena entidad.

\section{La dependencia de los hijos respecto del progenitor custodio}

El estudio realizado se centró fundamentalmente en el análisis de la monoparentalidad desde la perspectiva del progenitor, por lo que no han sido analizadas en su justa dimensión las características, experiencias y situaciones propias de los hijos. Como ya comentara Iglesias de Ussel, quizá en esta dimensión de dependencia se encuentra la base sobre la que se apoya una de las limitaciones que el término presenta, pues la expresión «familia monoparental»: «favorece la ocultación de los hijos y permite en la práctica situar en primer plano la dimensión de los adultos» (1998: 239).

El estudio de las familias monoparentales en Castilla y León puso en evidencia la importancia de considerar la dependencia de los hijos no tanto en términos de los años de éstos, sino también en función de las circunstancias que fuerzan la pervivencia de una dependencia del hijo respecto del progenitor en cualquiera de sus aspectos instrumentales. A efectos de la investigación llevada a cabo, la existencia o no de hijos dependientes en el seno del grupo familiar era libremente considerada por parte del progenitor, previa explicitación de los parámetros y las restricciones a la dependencia que ya han sido expuestos.

Entre los resultados más destacables habría que señalar, confirmando las tesis de Almeda y Flaquer (1995), la importancia que los padres y madres daban a la prolongada permanencia de los hijos en el sistema educativo y a las dificultades de empleo y de vivienda de los jóvenes para justificar la presencia en el hogar de hijos con más de 18 años, así como la gravedad de las situaciones de monoparentalidad en las que algún hijo presentaba algún tipo de minusvalía. Estas circunstancias llevaron a elevar la edad de dependencia potencial hasta los 26 años de edad, conscientes sin embargo de que el límite fijado tenía también un cierto carácter arbitrario.

\section{E. La posible convivencia del núcleo monoparental con otras personas}

Los datos obtenidos para Castilla y León corroboran la hipótesis de que muchos núcleos monoparentales recurren a la corresidencia con otras personas. 
Sin entrar aquí en las razones que explican este hecho (que sí son analizadas en el trabajo citado), hay que decir que sólo un $60 \%$ de los hogares monoparentales estudiados estaba constituido por un único núcleo monoparental. Las madres solteras tienden en mayor medida a vivir con otras personas, destacando la convivencia con los padres en casi la mitad de los casos. La corresidencia es menos frecuente en los casos de familias monoparentales como consecuencia de ruptura del vínculo de pareja y por viudedad.

El porcentaje tan significativo de núcleos monoparentales corresidiendo con otras personas es ya razón suficiente para rechazar la posibilidad de excluir del estudio de las familias monoparentales a las que no constituyen exclusivamente un hogar monoparental. Adicionalmente, la importancia del número de hogares en que el núcleo monoparental correside con terceras personas informa valiosamente de estrategias de convivencia a las que se recurre para resolver determinadas necesidades de organización de los miembros de estos grupos.

\section{F. La heterogeneidad en las causas que derivan en monoparentalidad}

Sin lugar a dudas, la diversidad es una de las características básicas que definen la naturaleza de la monoparentalidad. Diversidad de circunstancias que dan origen a un tipo u otro de monoparentalidad y diversidad en las experiencias, que dentro de una misma situación de monoparentalidad generará grupos bien diferenciados.

El estudio realizado sobre núcleos familiares monoparentales en Castilla y León confirmó los distintos procesos que derivan monoparentalidad y puso en evidencia la complejidad y pluralidad del fenómeno de la monoparentalidad. En el gráfico 1 se recogen los diferentes procesos y la importancia relativa de cada uno de ellos en Castilla y León. La tipología de familias monopa-

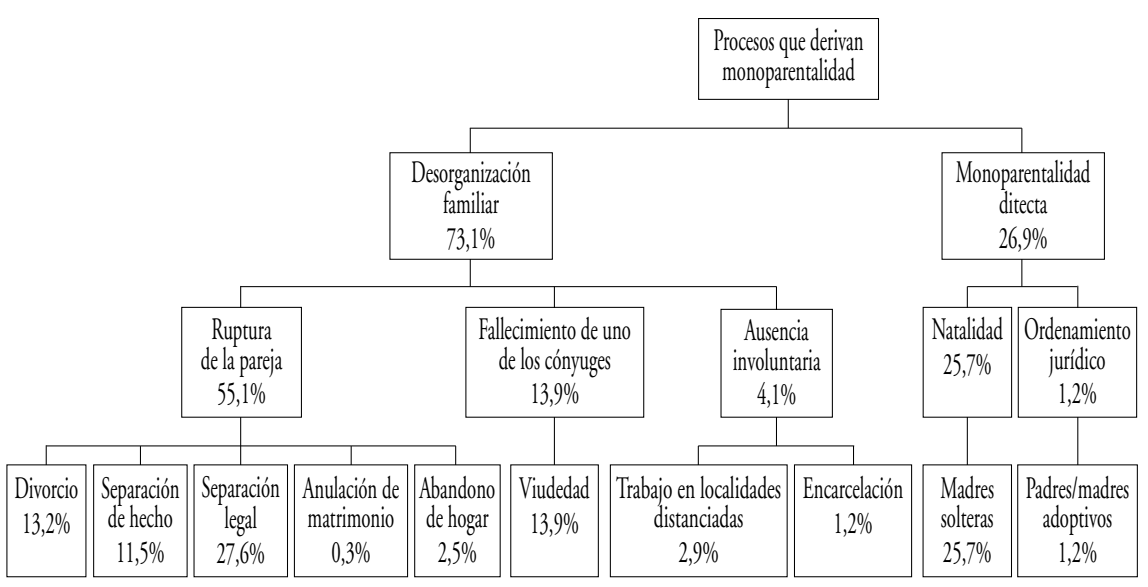

Gráfico 1. Procesos que derivan monoparentalidad y su importancia relativa en Castilla y León (\%). 
rentales resultante en virtud del proceso que da lugar a una reorganización familiar y que se presenta en dicho gráfico está inspirada en el modelo de Iglesias de Ussel (1988) y adaptada a la casuística castellano-leonesa.

A la luz de la investigación, dicha tipología, que había resultado ser útil como instrumento inicial de aproximación al fenómeno de la monoparentalidad, demostró constreñir en exceso el concepto de familia monoparental (y por tanto su análisis), al centrarlo esencialmente en la serie de procesos que determinan la reorganización de las estrategias familiares de los individuos.

\section{Consideraciones finales}

En estas líneas nos hemos ocupado de reflexionar sobre un concepto de familia monoparental que hacía especial referencia a los aspectos formales del grupo, por lo que nos hemos centrado en definir lo más operativamente posible los límites a partir de los cuales delimitar el fenómeno de la monoparentalidad con el objeto de poder estudiar su importancia y sus características en un ámbito geográfico determinado, en este caso Castilla y León.

Sin embargo, no podemos olvidar que, ante todo, la familia $-y$ por ende la familia monoparental - es una unidad no estática de relaciones que se configura dinámicamente en el tiempo y en el espacio. Esta consideración nos lleva a tener que reconocer la simplificación y deformación en las que incurrimos cuando tratamos de reducir a un concepto operativo una realidad tan compleja y multidimensional como es la que hemos pretendido analizar.

La contrastación de la definición operacional de familia monoparental de partida con la constelación de formas familiares cuya estructura formal se ajusta a los requisitos establecidos pone de relieve una cuestión fundamental: que los habituales parámetros utilizados para el análisis de la monoparentalidad pueden resultar insuficientes para captar la complejidad del fenómeno. Se puede decir así que existe toda una serie de dimensiones de la monoparentalidad cuya consideración obligaría no sólo a reconstruir el concepto, sino quizás incluso a reconsiderar su pertinencia. En este sentido, hemos ido señalando todo un conjunto de cuestiones que se plantean en torno a cada uno de los requisitos que vienen siendo habituales en la conceptualización de la monoparentalidad.

Hemos podido así comprobar que algunas de las condiciones que habitualmente se exigen a un grupo familiar para que éste pueda ser considerado como una familia monoparental plantean importantes interrogantes que nos llevan a confirmar el exclusivo valor instrumental que puede llegar a tener en ocasiones un concepto de partida. ¿Qué tienen verdaderamente en común una madre soltera adulta madura que buscó la maternidad fuera de la pareja y que goza de un estatus socioeconómico elevado, con una mujer en medio de un proceso complejo de separación y sin recursos económicos ni apoyo de su entorno? ¿ No podría ser ciertamente considerado como monoparental el conjunto formado por un progenitor y sus hijos aunque formen parte de una estructura familiar nuclear conyugal cuando ocurre que el otro progenitor no ejerce funcionalmente como tal? ¿Hasta qué punto es válido hablar de ausen- 
cia de un progenitor en el caso de las familias separadas o divorciadas donde ambos cónyuges comparten sus tareas de atención y educación de los hijos aunque éstos corresidan la mayor parte del tiempo con uno de ellos? ¿Hasta qué punto podemos también hablar de ausencia de un progenitor cuando el grupo familiar percibe que la ausencia física (sobre todo cuando es temporal) no impide el ejercicio de las funciones de paternidad, o cuando estas funciones las cumple otra persona diferente al progenitor?

El análisis practicado pone de manifiesto que la tipología de familias monoparentales que subyace al concepto operacional de partida y que clasifica a éstas en virtud del proceso que desemboca en reorganización familiar debemos entenderla tan sólo como el resultado de una categorización social. En otras palabras, tiene valor en tanto que «ayuda para interpretar situaciones ambiguas mediante la acentuación de las diferencias entre categorías y la homogeneidad dentro de la categoría» (Morales, 1999: 209), pero no confiere al objeto de estudio mayor entidad, al ser tan sólo un instrumento útil para el observador. Resulta por tanto necesario profundizar en el conocimiento de la naturaleza dinámica de la monoparentalidad, en la línea de los análisis realizados para el Reino Unido por Ermish, Jenkins y Wright (1990) y Noble, Smith y Cheung (1998) ya citados.

A partir de la investigación llevada a cabo se observó que el concepto y la tipología de partida presentaban, al menos, tres virtudes fundamentales. En primer lugar, representaban una válida categorización de los factores que modulan situaciones de monoparentalidad y, por tanto, de la propia dinámica social. En segundo lugar, facilitaban la interpretación de realidades complejas y plurales que, aún teniendo orígenes comunes, elaboran estrategias de convivencia diferentes y se nutren de contenidos que faciliten identidades únicas. Así, cada uno de los grupos familiares estudiados se presentaban como microsociedades en las que aparecían claramente diferenciadas sus economías, culturas, hábitos y la propia percepción de su situación de monoparentalidad. Finalmente, resultaron ser un buen punto de partida para la instrumentación estadística.

Asimismo, la investigación evidenció también algunos riesgos derivados del recurso a la categorización. En primer lugar, se comprobó que sienta las bases para el funcionamiento de los estereotipos y puede actuar como mediador cognitivo excluyente. En este sentido, los resultados del trabajo con adultos al frente de núcleos familiares monoparentales, en la línea de lo confirmado por Fry y Addington $(1984)^{3}$, detectaron la presencia de estereotipos sociales con relación a las familias monoparentales. Casi cuatro de cada diez madres y padres informaron sentirse discriminados por el hecho de pertenecer a una familia considerada por terceros como no estándar. En segundo lugar, se comprobó que el concepto de partida mantenía ocultos elementos fundamentales para la vida de estos padres y madres y sus familias, tales como la red compleja de parentesco que en muchas de las situaciones intensifican su apoyo ins- 
trumental y/o afectivo a las personas residiendo en núcleos familiares monoparentales. Por último, resultó evidente que las categorizaciones sociales tienen un valor exclusivamente metodológico y, además, limitado. En otras palabras, diluir el concepto de familia en las causas que lo modulan, es adoptar una visión reduccionista y estática del grupo familiar. Otras dimensiones, tales como la representación de la familia que tiene una sociedad, la idea de divorcio y el cuerpo legal que una sociedad posee, la representación social de la responsabilidad del cuidado de la infancia, además de las actitudes ante la maternidad fuera del matrimonio, entre otros, son contenidos a tener necesariamente en cuenta a la hora de definir la monoparentalidad y estudiar cualquiera de sus manifestaciones, así como a la hora de diseñar y poner en práctica estrategias de intervención sobre el fenómeno.

El trabajo que aquí se presenta se apoya metodológicamente en la reconstrucción de un proceso de investigación, en la exposición de su historia interna. Somos conscientes de que la propuesta lakatosiana de elaborar reconstrucciones racionales contiene sin embargo, como recuerda Medina (1989: 112), toda "una serie de inconvenientes de entre los cuales el idealismo y la arbitrariedad no son los menores». Es por ello que se ha intentado hacer una revisión crítica del proceso y presentar con claridad y transparencia lo que el contacto con los padres y madres al frente de un núcleo monoparental nos informó sobre la validez del concepto de partida.

Es cierto que no es posible pensar sin conceptos o modelos, y que éstos tienen la virtud de orientarnos a la realidad. Pero no es menos cierto que los conceptos que manejamos tienen un coste en términos de distorsión de esa misma realidad, de adecuación de la misma a los ojos del observador. Éstos son algunos de los problemas sobre los que hemos pretendido reflexionar en nuestro artículo en un intento de contribuir, cuando menos, a la discusión y al análisis del concepto de familia monoparental.

\section{Bibliografía}

AlberDi, I. (1988). «Las mujeres viudas y las familias monoparentales», en J. IGLESIAS DE UsSEL (ed.). Las familias monoparentales, Madrid: Ministerio de Asuntos Sociales, Instituto de la Mujer, Serie Debate, núm. 5, p. 99-108.

Almeda, E.; Flaquer, L. (1995). «Las familias monoparentales en España: un enfoque crítico", Revista Internacional de Sociología, núm. 11, p. 21-45.

Almodóvar, J. P. (1988). "El niño, la infancia, los niños y el divorcio», en J. IGLESIAS DE USSEL (ed.). Las familias monoparentales, Madrid: Ministerio de Asuntos Sociales, Instituto de la Mujer, Serie Debate, núm. 5, p. 177-192.

BORRAJO INIESTA, S. (1988). "Ruptura matrimonial y formación de familias monoparentales en España", en J. IGLESIAS DE USSEL (ed.). Las familias monoparentales, Madrid: Ministerio de Asuntos Sociales, Instituto de la Mujer, Serie Debate, núm. 5, p. 41-48.

COMISIÓN DE DeRECHOS DE LA MuJer (1998). Informe sobre la situación de las madres solas y las familias monoparentales, Parlamento Europeo, Informe A4-0273/98, de 9 de julio. 
COMISIÓN EUROPEA (1982). Las familias monoparentales y la pobreza, Comisión Europea, DOC V/2541/1/82.

- (1989). Lone Parent Families in the European Community: Final Report, Londres: Family Policy Studies Center.

CONSEJO DE EUROPA (1995). Evolución demográfica reciente en Europa 1995, Consejo de Europa.

Delgado, M. (1994). La fecundidad de las adolescentes, Madrid: CIS.

DUNCAN, G. y otros (1994). "Les familles monoparentales aux États-Unis. Dynamique, niveau de vie et conséquences sur le developpement de l'enfant», Population, 6, p. 1419-1436.

DURÁN, M. A. (1988). «Hogares y familias: dos conceptos en busca de definición», en J. IgLESIAS DE USSEL (ed.). Las familias monoparentales, Madrid: Ministerio de Asuntos Sociales, Instituto de la Mujer, Serie Debate, núm. 5, p. 11-22.

ERMiSH, J. F.; JENKINS, S.; Wright, R. E. (1990). «Analysis of the dynamics of lone parenthood: socioeconomic influencies on entry an exit rates», en E. DUSKIN (ed.). Lone parent families: the economic challenge, Social Policy Studies, núm. 8, p. 69-90.

ESRC (1994). Changing households: the British Household Panel Survey 1990-1992, Colchester, ESRC Research Centre o Micro-social Change.

FERnÁNDEZ Cordón, J. A.; Tobío soler, C. (1998). «Las familias monoparentales en España», Revista de Investigaciones Sociológicas, núm. 83, p. 51-85.

- (1999). Las familias monoparentales en España, Madrid: MTAS, Colec. Estudios, núm. 34.

FORD, R.; MARSH, A.; McKAY, S. (1995). Changes in lone parenthood, Londres: HMSO.

IGLESIAS DE USSEL, J. (1988a). «La situación de la familia en España y los nuevos modelos familiares», en J. IGLESIAS DE USSEL (ed.) Las familias monoparentales, Madrid: Ministerio de Asuntos Sociales, Instituto de la Mujer, Serie Debate, núm. 5, p. 23-40.

- (ed.) (1988b). Las familias monoparentales, Madrid: Ministerio de Asuntos Sociales, Instituto de la Mujer, Serie Debate, núm. 5.

- (1998). La familia y el cambio político en España, Madrid: Tecnos.

LAMO DE EsPINOSA, E. (1990). La sociedad reflexiva. Sujeto y objeto del conocimiento sociológico, Madrid: CIS, Colec. Monografías, núm. 144.

LE GALL, D.; MARTIN, C. (1988). «El ámbito de sociabilidad del niño en los hogares monoparentales de divorciados y separados», en J. IGLESIAS DE USSEL (ed.). Las familias monoparentales, Madrid: Ministerio de Asuntos Sociales, Instituto de la Mujer, Serie Debate, núm. 5, p. 195-202.

LEFAUCHEUR, N. (1988). «¿Existen las familias monoparentales?», en J. IGLESIAS DE UsSel (ed.). Las familias monoparentales, Madrid: Ministerio de Asuntos Sociales, Instituto de la Mujer, Serie Debate, núm. 5, p. 153-162.

LEWIS, J. (1999). "The "problem" of lonemotherhood in comparative perspective», en J. ClANSEN (ed.). Comparative social policy: concepts, theories and methods, Oxford: Blackwell, p. 181-199.

Madruga, I.; Mota, R. (1999). Las condiciones de vida de los hogares pobres encabezados por una mujer. Pobreza y género, Madrid: Cáritas y Fundación Foessa.

MARTIN, C. (1994). «Diversité des trajectoires post-union. Entre le risque de solitude, la défense de son autonomie et la récomposition familiale», Population, 6, p. 1557-1583.

MCINTOSH, M. (1996). «Social anxieties about lone motherhood and ideologies of the family: two sides of the same coin», en E.B. Silva (ed.) Good enough mothering? Feminist perspectives on lone motherhood, Londres: Routledge, p. 148-156. 
Medina, E. (1989). Conocimiento y sociología de la ciencia, Madrid: CIS, Colec. Monografías, $n^{\circ} 107$.

MORA, G. (2001). «Las familias españolas ante el cambio de siglo», en VVAA. Estructura y cambio social. Libro homenaje a S. del Campo, Madrid: CIS, p. 293-312.

Morales, J. F.; OlZA, M. (coord.) (1999). Psicología social y trabajo social, Madrid: McGraw-Hill.

Moreno Hernández, A. (1995). «Familias monoparentales», Infancia y Sociedad, núm. 30, p. 56-64.

Moreno Mínguez, A. (2000). "Las familias monoparentales en España», Revista Internacional de Sociología, núm. 26, p. 39-63.

Noble, M.; SMITH, G.; CHEUnG, S. Y. (1998). «Lone mothers moving in and out of benefits», Work and Opportunity Series, núm. 4, York: Joseph Rowntree Foundation.

Rodríguez SumAZA, C. (2001a). "Lone parent families within New Labour welfare reform», Contemporary Politics, vol. 7 (3), p. 231-247.

- (2001b). «La estrategia británica de apoyo a las familias monoparentales a través del empleo", Revista Internacional de Sociología, núm. 30, p. 209-239.

RodrígueZ SumAZA, C. y LuENGo Rodríguez, T. (dirs.) (2000). Las familias monoparentales en Castilla y León, Valladolid: Junta de Castilla y León, Consejería de Sanidad y Bienestar Social.

Roll, J. (1992). «Familias monoparentales en Europa», Infancia y Sociedad, núm. 16, julio-agosto, p. 155-170.

Ruiz Olabuenaga, J. I.; Aristegui, I.; Melgosa, L. (1988). Cómo elaborar un proyecto de investigación social, Bilbao: ICE Universidad de Deusto.

SAYN, I. (1988). «Las familias monoparentales: Derecho Civil y Derecho Social», en J. IgLeSIAS DE UsSEL (ed.). Las familias monoparentales, Madrid: Ministerio de Asuntos Sociales, Instituto de la Mujer, Serie Debate, núm. 5, p. 203-210.

TAYLOR, S. J.; GOGDAN, R. (1994). Introducción a los métodos cualitativos de investigación, Barcelona: Paidós.

WEBER, M. (1969). Economía y sociedad. Esbozo de sociología comprensiva, México: Fondo de Cultura Económica, vol. 1. 